\title{
Novel stiffeners exploiting internal pressurisation to enhance buckling behaviour under bending loads
}

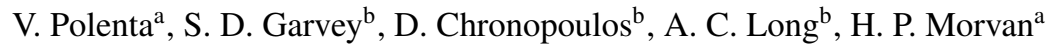 \\ ${ }^{a}$ Institute for Aerospace Technology, Aerospace Technology Centre, The University of Nottingham, Nottingham NG7 2TU, United Kingdom \\ ${ }^{b}$ Faculty of Engineering, University Park, The University of Nottingham, Nottingham NG7 2RD, United Kingdom
}

\begin{abstract}
The paper proposes a novel type of stiffener designed to bear bending loads by exploiting internal pressure effects. The stiffener is made of two adjacent thin-walled pipes $(r / t \geq 50)$ jointed with a connecting strip. Such structure is shown to have higher performance against buckling failure compared to a single pipe and its geometry allows for good exploitation of internal pressurisation.

The study is conducted by using the FEA software ANSYS and the analysis technique is the linear perturbation buckling analysis. Internal pressure ranges from 0 to $1.4 \mathrm{MPa}$. The buckling mechanisms are observed for a set of models with different values of length, wall thickness and geometric variation of the cross-section. It is shown that two different buckling modes can take place. However, for a given geometry, the level of pressure can alter the behaviour and lead to one mode rather than the other one.

Potential of the presented structure is maximised by the use of high performance materials and a possible aerospace engineering application is presented.
\end{abstract}

Keywords: Buckling, Internal pressure, Pure bending, Thin-walled shell

\section{Introduction}

Many engineering applications see the use of slender parts and thin-walled components. These allow limiting the amount of material and consequently the weight whilst providing structural strength and rigidity. On the other hand, design of slender parts and thin-walled components has to keep in consideration that they tend to exhibit structural stability problems, i.e. buckling failure. Therefore extensive studies have been made on this matter.

The first studies on buckling were carried out in XVIII century by Euler who solved the problem of lateral buckling of compressed members and further research was made starting from the XIX century [1, 2]. Most of the relevant literature dates back to the XX century, when many authors investigated on buckling of thin cylindrical shells $[3,4,5]$. It was found that the buckling behaviour of cylindrical shells subjected to bending depends on the material parameters as well as geometric parameters, especially the diameter-thickness ratio $D / t$ [6]. Thick wall pipes $(D / t<40$ for steel) exhibit a plastic buckling [7]. On the other hand, thin wall pipes $(D / t>100$ for steel $)$ buckle at first in the elastic region. Thus, the buckling load depends by some material parameters (Young's Modulus $E$ and Poisson ratio $v$ ) but not by the material yield stress $\sigma_{y}$. Some authors $[2,3,8]$ conducted studies on the elastic buckling of circular pipes under bending and their results of the buckling load differ significantly within a wide range of values. The reason of this variability is that the behaviour (and the strength) of circular pipes is strongly depending on other factors other

Email address: valerio.polenta@nottingham.ac.uk (V. Polenta) than material properties, such as diameter-thickness ratio $D / t$, constraints and cross-section flattening.

Authors proved that internal pressure increases the limit buckling load of thin cylindrical shells for different load conditions such as axial load [9], torsion [10] and bending [11, 12]. In the case of bending, benefits of pressure are not only related to the stabilisation effect but also to an anti-flattening effect which decreases the cross-section inertial moment reduction and, therefore, the stress in the bent pipe [13]. It was also proved that pipe curvature has effects on buckling phenomenon and curved pipes buckle earlier than the corresponding straight pipes [14], [13] and [12].

In our previous work [15] we showed that, for a given pipe, there exists an optimal pressure to which buckling failure and yield failure have the same limit. This allows to fully exploit the bearing capacity of the pipe and maximise its limit bending load. Therefore, we proposed an optimisation of the pipe based on internal pressure and pipe curvature. Now we carry on with maximising limit load for thin-walled cylinders by studying a slightly more complex geometry made of two adjacent pipes firmly connected each other by a strip. Such stiffener, that we will be referring to it as "pinched pipe", is subjected to internal pressure and bending moment and its buckling behaviour is studied by FEA. We show that for a certain pipe radius $r$ and length $L$, the pinched pipe exhibits higher buckling load over weight ratio than the single pipe. Moreover, the geometry of the pinched pipe still allows to internally pressurise the structure and exploit the consequent benefits. This structure reveals high potential in application wherein load withstanding capacity is needed.

The paper is organised as follow: Section 2 describes the 


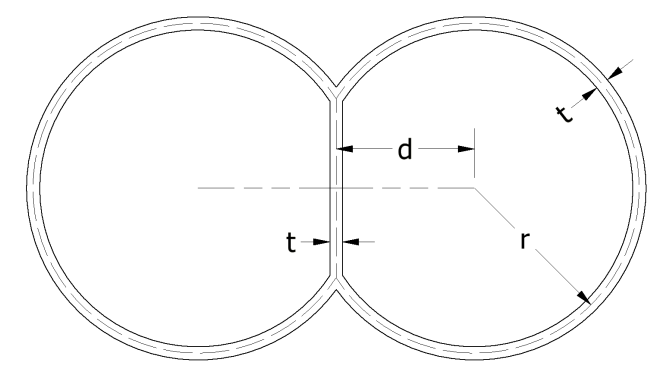

Figure 1: Cross-section geometry and main geometric parameters: radius $r$, semi-distance $d$ and wall thickness $t$.

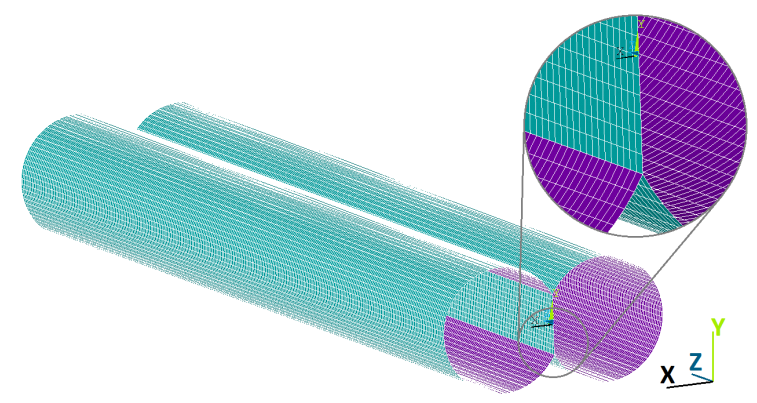

Figure 2: FE model. Only half span of the pinched pipe is modeled by taking advantage of the symmetry.

FE model; Section 3 explicates the analysis procedure; Section 4 is divided in four subsections presenting the numerical results; Section 5 discusses the obtained results; Section 6 provides some remarks on the applications and feasibility of the presented structure; Section 7 concludes the paper.

\section{FE model}

The FEA software used to perform buckling analysis is ANSYS 15.0. The model geometry consists in a right-prismatic shape. Three surfaces can be identified: a middle plate, which will be referred to as "strip", and two adjacent and identical cylindrical shells symmetrical with respect to the strip and connected to the latter along the edges. The entire structure is referred to as pinched pipe. Fig. 1 shows the cross-section geometry with the relevant geometric parameters; Fig. 2 shows the meshed model and reference system.

The problem is modeled with SHELL181 which is a fournodes shell element. The mesh size, based upon a sensitivity study, is set to $3 \mathrm{~mm}$ along the axial direction and approximately $4 \mathrm{~mm}$ along the hoop direction (corresponding to 0.06 and 0.08 times the radius $r$, respectively). Bending moments around the $\mathrm{X}$ axis are applied to the nodes of the two stiffener ends, rotating them downwards. The ends are constraints to behave as rigid planes. The other acting load is pressure applied to the inner surfaces. The material model is linear and isotropic with typical properties of a steel. In order to reduce the computational power required by the simulations only half of the pinched pipe is modeled taking advantage of the symmetry of
Table 1: FE model parameters

\begin{tabular}{llc}
\hline \hline Parameter & Type & Value \\
\hline Pipe radius, $r$ & fixed & $50 \mathrm{~mm}$ \\
Pipes semi-distance, $d$ & variable & {$[40 \mathrm{~mm}, 48 \mathrm{~mm}]$} \\
Length, $L$ & variable $^{1}$ & {$[600 \mathrm{~mm}, 2200 \mathrm{~mm}]$} \\
Wall thickness, $t$ & variable & $0.25 \mathrm{~mm}, 0.50 \mathrm{~mm}, 1.00 \mathrm{~mm}$ \\
Internal pressure, $p$ & variable & {$[0 \mathrm{MPa}, 1.4 \mathrm{MPa}]$} \\
Young Modulus, $E$ & fixed & $200 \mathrm{GPa}$ \\
Poisson's ratio, $v$ & fixed & 0.3
\end{tabular}

the problem. Therefore, additional constraints are added to the XY plan (middle plane along the span).

Table 1 summarises the model parameters.

\section{The analysis}

The FE simulations hereby conducted aim to quantify the limit buckling moment for the different geometric parameters and values of internal pressure.

As shown in our previous work [15], linear and non-linear analysis can lead to somewhat different results. Linear buckling analysis is essentially an eigenvalue problem. Its formulation is the following [16]:

$$
\left(\mathbf{K}+\lambda_{j} \mathbf{S}\right) \psi_{j}=\mathbf{0}
$$

where $\mathbf{K}$ is the stiffness matrix, $\mathbf{S}$ is the stress stiffness matrix, $\lambda_{j}$ is the $j^{\text {th }}$ eigenvalue (used to multiply the loads which generated $\mathbf{S}$ ) and $\psi_{j}$ is the $j^{\text {th }}$ eigenvector of displacements. The simulation time is generally short and more eigenvalues and the corresponding eigenvectors can be obtained in order to predict what the buckling modes will be like; on the other hand, it might over-estimate the actual buckling load.

Non-linear buckling analysis is a static analysis wherein large deflections are accounted for. Loads are applied in gradual steps to seek the load level at which the structure becomes unstable. The problem is solved with the Newton-Raphson method, an iterative process use to solve non-linear equations. In a structural analysis, the problem formulation is the following:

$$
\begin{gathered}
\mathbf{K}_{\mathrm{i}}^{\mathbf{T}} \Delta u_{i}=F^{a}-F_{i}^{n r} \\
u_{i+1}=u_{i}+\Delta u_{i}
\end{gathered}
$$

where the subscript $i$ represents the current iteration, $\mathbf{K}_{\mathbf{i}}^{\mathbf{T}}$ is the tangent stiffness matrix, $\boldsymbol{u}_{\boldsymbol{i}}, \boldsymbol{u}_{\boldsymbol{i}+\mathbf{1}}$ and $\Delta \boldsymbol{u}_{\boldsymbol{i}}$ represent vectors of degree of freedom values, $\boldsymbol{F}^{a}$ is the vector of applied loads and $\boldsymbol{F}_{\boldsymbol{i}}^{\boldsymbol{n} \boldsymbol{r}}$ is the vector of restoring loads corresponding to the element internal loads. Non-linear analysis is more accurate but presents some disadvantages. The main ones are the longer computational time and, in case of buckling, the need to trigger the instability by means of geometric imperfections and/or perturbation loads.

\footnotetext{
${ }^{1}$ The problem is mainly investigated with a model $1600 \mathrm{~mm}$ long although different values are analysed to study the influence of length.
} 

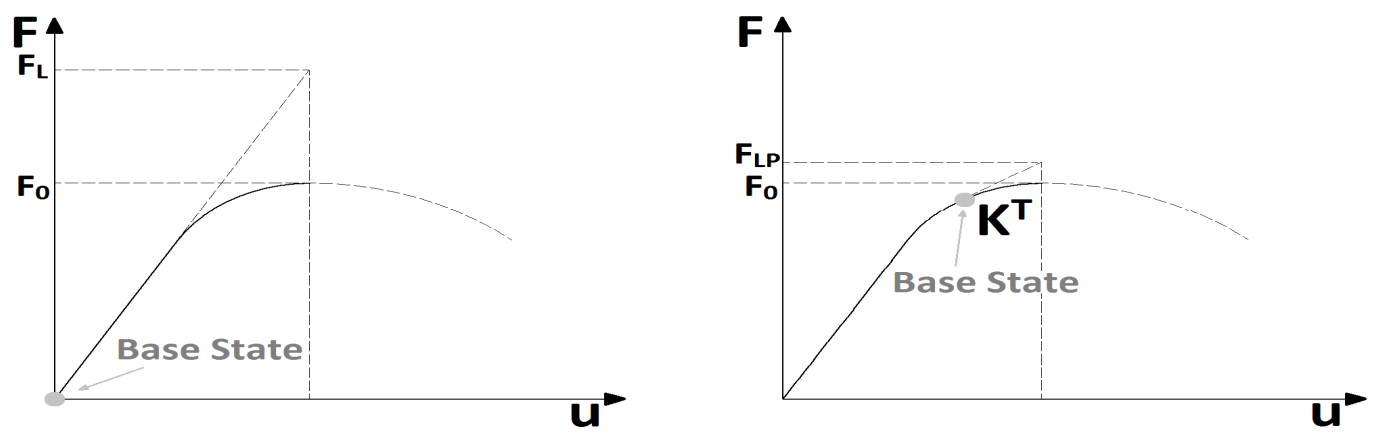

Figure 3: (a) Conventional linear buckling analysis: $F_{L}$ is the predicted buckling load, $F_{0}$ is the actual buckling load. $(b)$ Linear perturbation buckling analysis: the preloaded base state and the corresponding tangent stiffness matrix $K^{T}$ allow predicting a more accurate buckling load $F_{L P}$.

Hereinafter we adopt a different analysis technique to overcome the limitations of the two techniques above. The ANSYS theory refers to such analysis procedure as linear perturbation buckling analysis. It consists in two phases: (i) a non-linear static analysis up to a point prior to buckling; (ii) a linear buckling analysis (eigenvalue problem) starting from the preloaded condition calculated in the first phase [17]. Between phase (i) and phase (ii) a restart procedure is performed.

This allows the eigenvalue problem to be based on the global tangent stiffness matrix $\mathbf{K}^{\mathbf{T}}$, updated after each step of the Newton-Raphson procedure of phase (i), rather than the linear stiffness matrix, as schematically shown in Fig. 3. The global tangent stiffness matrix, which is used in the eigenvalues calculation of phase (ii), can be segregated into matrices as follows:

$$
\mathbf{K}^{\mathrm{T}}=\mathbf{K}^{\mathrm{M}}+\mathbf{S}^{\mathrm{N}}+\mathbf{K}^{\mathrm{LD}}+\mathbf{K}^{\mathrm{C}}+\mathbf{K}^{\mathrm{SP}}
$$

where $\mathbf{K}^{\mathbf{M}}$ is the part of the tangent stiffness contributed from the material property, $\mathbf{S}^{\mathbf{N}}$ is the stress stiffening matrix introduced by non-zero stresses, $\mathbf{K}^{\mathbf{L D}}$ is the load stiffening matrix introduced by external pressure loads or by follower force effect, $\mathbf{K}^{\mathbf{C}}$ is the total stiffness matrix contributed from contact elements of the model and $\mathbf{K}^{\mathbf{S P}}$ is the spin-softening matrix introduced by rotational velocities. The load applied in phase (i) is referred to as $\boldsymbol{F}_{\text {restart }}$; the load (generally unitary) applied in phase (ii), after the restart procedure, is referred to as $\boldsymbol{F}_{\text {perturbed }}$. The latter is used to calculate the linearly perturbed displacement $\boldsymbol{U}_{\text {perturbed }}$ :

$$
\mathbf{K}_{\mathrm{i}}^{\mathrm{T}} \boldsymbol{U}_{\text {perturbed }}=\boldsymbol{F}_{\text {perturbed }}
$$

$\boldsymbol{U}_{\text {perturbed }}$ is used to calculated the linearly perturbed stresses and the linearly perturbed stress stiffening matrix $\mathbf{S}_{\text {perturbed }}$. The eigenvalue problem can then be solved:

$$
\mathbf{K}_{\mathbf{i}}^{\mathbf{T}} \psi_{j}=\lambda_{j} \mathbf{S}_{\text {perturbed }} \psi_{j}
$$

Eq. (6) is mathematically equal to Eq. (1) but uses matrices based on the current load condition to calculate eigenvalues and eigenvectors.

The final step of the analysis consists in calculating the final buckling load $\boldsymbol{F}_{\text {buckling. }}$. If $\lambda_{1}$ is the lowest eigenvalue calculated with Eq. (6), the final buckling load is given by

$$
\boldsymbol{F}_{\text {buckling }}=\boldsymbol{F}_{\text {restart }}+\lambda_{1} \boldsymbol{F}_{\text {perturbed }}
$$

The linear perturbation buckling analysis as described above requires that loads applied in phase (i) $\left(\boldsymbol{F}_{\text {restart }}\right)$ are lower the buckling load (to avoid convergence issues) but as close as possible to the latter. As highlighted by Fig. 3, a restart point close to the buckling point leads to higher accuracy. Thus, iterations with a increasingly $\boldsymbol{F}_{\text {restart }}$ are needed. In order to have high accuracy, the results presented in the next section were obtained with analyses wherein $\boldsymbol{F}_{\text {restart }}$ is at least $95 \%$ of $\boldsymbol{F}_{\text {buckling }}$.

\section{Numerical results}

\subsection{Length sensitivity}

An initial set of simulations studied the effect of the length of the pinched pipe. In particular, the aim was to identify the length which allows buckling load not being affected by the ends constraints. In this conditions, results can be generalised to longer models.

Effects of boundary conditions on cylindrical shells under bending have been studied by several authors and the axial length of pipe affected by the end constrains cannot be easily established a priori [18]. In order to study the behaviour of the shell under pure bending, the most effective way of modeling is to leave the pipe's ends free to ovalise while remaining planar. Alternatively, the model can include constrains that fully restrain the end deformation provided that the pipe length is adequate, i.e. long enough for the constraint effects to vanish in the mid region of the pipe. This simply consists in taking advantage of the De-Saint-Venant's Principle [19]. In the present paper, the latter solution was chosen, as described in Section 2. Generally, this solution is not optimal since it requires a longer model and consequently more computational power is needed. However such approach enabled comparison with previous work [15].

Simulations were performed on models of radius $r=50 \mathrm{~mm}$, wall thickness $t=0.25$ and $t=1.00 \mathrm{~mm}$, internal pressure $p=0$ and $p=1.4 \mathrm{MPa}$, pipe semi-distance $d=40$ and $d=48$ $\mathrm{mm}$ and different values of length $L$. The three pairs of values for $t, p$ and $d$ are the upper and lower bounds of the parameters 


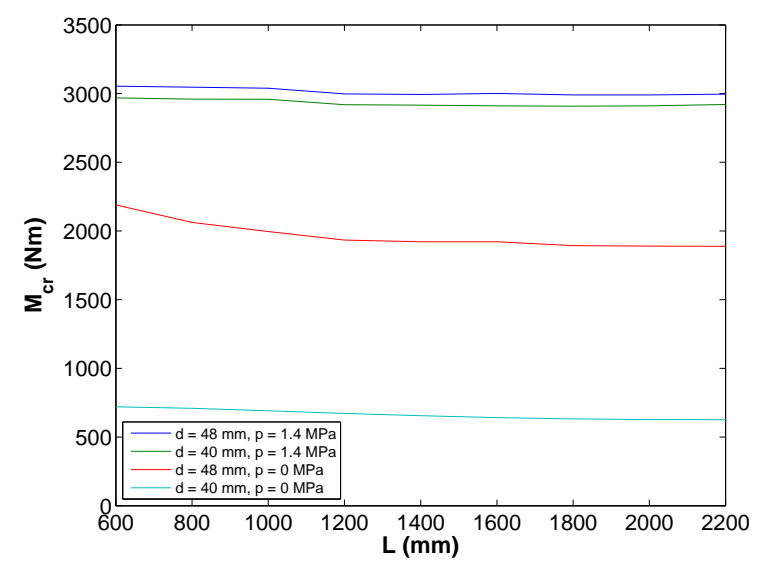

Figure 4: Critical moment $M_{c r}$ as a function of pipe length $L$. Wall thickness $t=0.25 \mathrm{~mm}$.

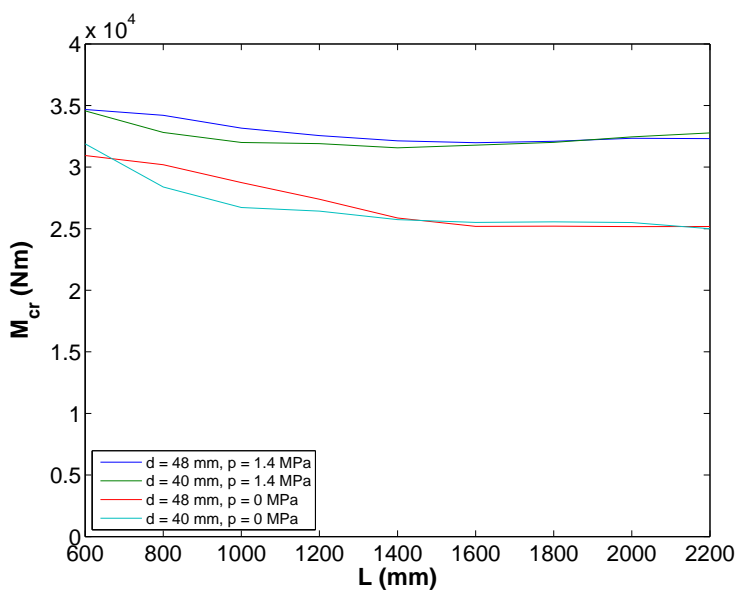

Figure 5: Critical moment $M_{c r}$ as a function of pipe length $L$. Wall thickness $t=1.00 \mathrm{~mm}$.

listed in Table 1 in order to have the length sensitivity study representative of the all set of models analysed in the present work. Fig. 4 and Fig. 5 show the critical moment $M_{c r}$ as a function of pipe length $L$ for pinched pipes with wall thickness $t=0.25$ and $t=1.00 \mathrm{~mm}$, respectively.

It can be observed that critical moment decreases with pipe length. It was expected since pipes ends are constrained to behave as rigid planes: for short pipes, end effects influence the mid-section and this helps to prevent buckling. $1600 \mathrm{~mm}$ is found to be the representative length for the range of analysed models.

In order to further validate the outcome of the length sensitivity study represented by Figs. 4 and 5 , an additional investigation was performed in order to obtain not only the critical moment, but also the ovalisation $\theta$ as a function of pipe length. Pipe ovalisation is generally defined as

$$
\theta=\frac{D_{\max }-D_{\min }}{D}
$$

where $D_{\text {max }}, D_{\min }$ and $D$ are the maximum, minumum and nominal diameter, respectively. However, due to the peculiar cross-

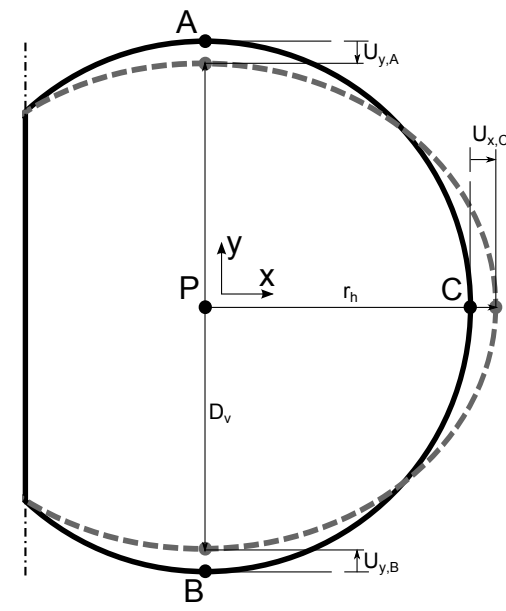

Figure 6: Rapresentation of pinched pipe deformation: the initially perfect cylindrical shell (black pipe) deforms into the ovalised shape (grey dashed pipe). Only half structure is shown due to symmetry.

section of the pinched pipe, ovalisation cannot be simply defined as in Eq. 8. With a small extent of simplification, ovalisation can be evaluated by considering the displacements of three specific points (or nodes), referred to as points A, B and $\mathrm{C}$ in Fig. 6. The symmetry of the pinched pipe with respect to the middle strip is taken into account therefore only half of the structure is considered.

Naming $D$ the initial diameter, $D_{v}$ the deformed vertical diameter, $r$ the initial radius, $r_{h}$ the deformed horizontal radius, $U_{y, A}, U_{y, B}$ and $U_{x, C}$ the displacements of the points, similarly to Eq. 8 one has

$$
\begin{array}{r}
\theta=\frac{2 r_{h}-D_{v}}{D}=\frac{2 r_{h}-D+D-D_{v}}{D}= \\
=\frac{2\left(r+U_{x, C}\right)-2 r+D-\left(D+U_{y, A}-U_{y, B}\right)}{D}= \\
=\frac{2 U_{x, C}-U_{y, A}+U_{y, B}}{D}
\end{array}
$$

Eq. 9 provides a simple expression for the pinched pipe ovalisation and assumes that points $\mathrm{A}$ and $\mathrm{B}$ mainly move in the $\mathrm{Y}$ direction and point $\mathrm{C}$ mainly move in the $\mathrm{X}$ direction.

Fig. 7 shows both the critical moment and ovalisation as functions of pipe length for a certain pinched pipe model. Ovalisation is calculated based on Eq. 9. It is evaluated at the midsection at the end of the nonlinear linear step, i.e. right before performing the eigenvalue buckling analysis. It was found that ovalisation increases as the pipe length increases. Furthermore, as for the critical moment, the ovalisation curve trend is asymptotic, i.e. maintains a constant value for long pipes. Such longenough condition is consistent for critical moment and ovalisation. The two physical quantities reach the quasi-asymptotic behaviour at the same value of length (within a certain tolerance), confirming that critical moment and mid-section ovalisation are in relationship. Again, $1600 \mathrm{~mm}$ is found to be the representative length and all the following results will refer to models with such length. 


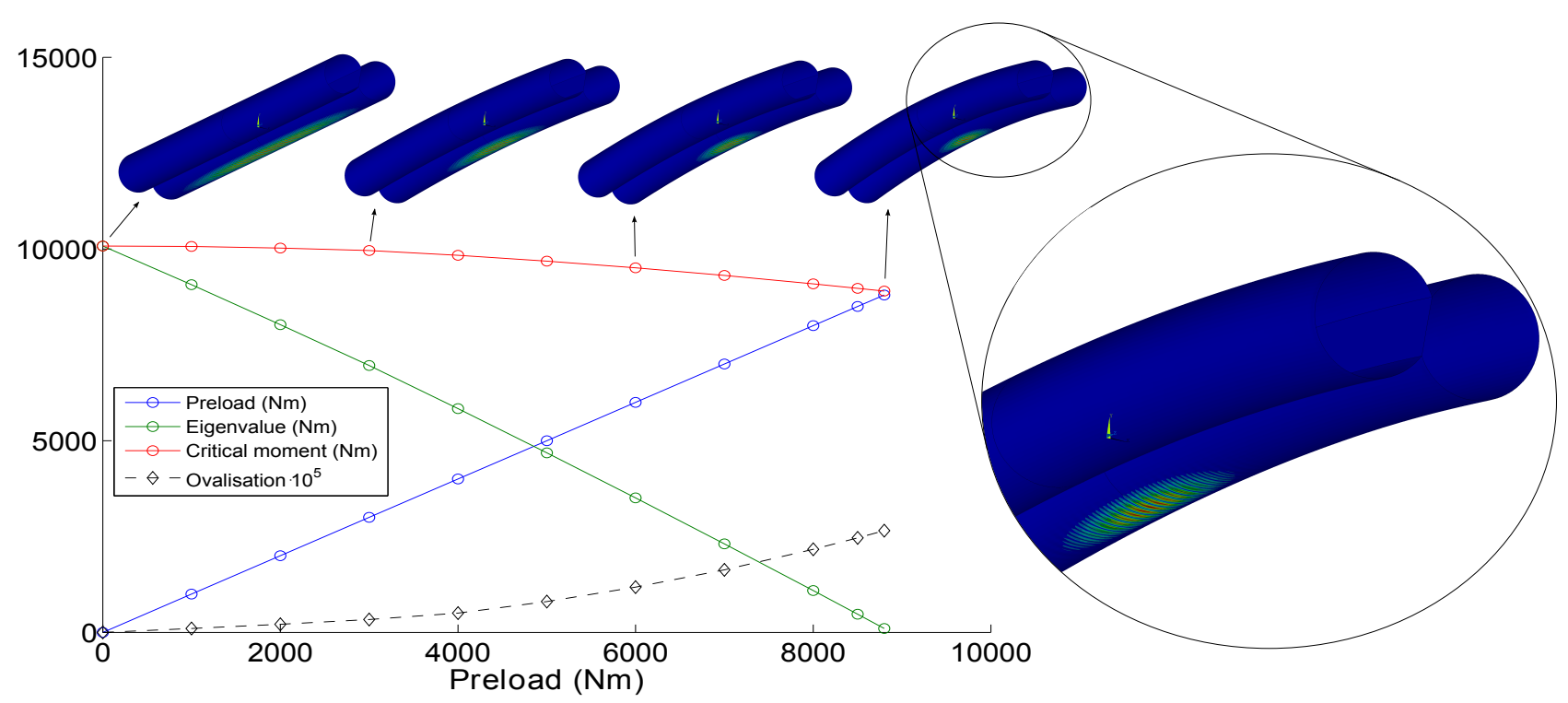

Figure 8: Effects of the preload on a pinched pipe with length $L=1600 \mathrm{~mm}$, wall thickness $t=0.50 \mathrm{~mm}$, pipe semi-distance $d=44 \mathrm{~mm}$ and internal pressure $p=0.5 \mathrm{MPa}$.

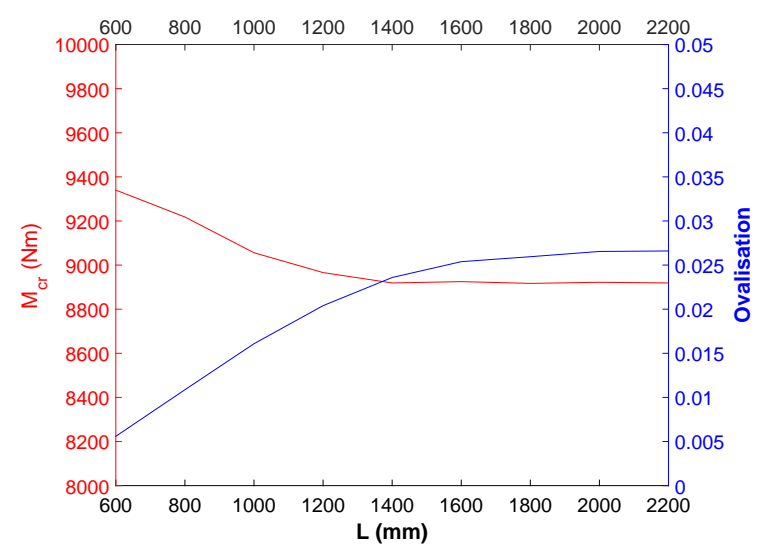

Figure 7: Critical moment $M_{c r}$ and mid-section ovalisation $\theta$ as functions of pipe length $L$. Wall thickness $t=0.50 \mathrm{~mm}$, pipe semi-distance $d=44 \mathrm{~mm}$ and internal pressure $p=0.5 \mathrm{MPa}$.

Finally, it is interesting to note that the ends of a pinched pipe have a longer zone of influence than the ones of single pipes. For $r / t$ ranging from 50 to 200 , a length corresponding to $L / r \approx$ 16 is enough to consider a single pipe long $[13,15]$. In other terms, for $L / r \geq 16$ buckling is no more affected by the pipe length. On the other hand, a pinched pipe needs to be longer in order that the mid-section is not affected by ends constraints, due to its wider cross-section geometry. Figs. 4, 5 and 7 show that critical moment is no longer affected by ends constraints for values of length grater than $1600 \mathrm{~mm}$, corresponding to $L / r=$ 32.

\subsection{Effect of preload on pinched pipe}

As described in Section 3 and by Fig. 3, the analyses here performed consist of two phases. The first phase preloads the structure. Here the effects of the preload is investigated.
Fig. 8 shows the analysis results for a certain model and different levels of preload. Specifically, each linear perturbation buckling analysis is performed for a specific level of preload to be applied in phase (i) and provides an eigenvalue as result of phase (ii). Ultimately, the critical buckling load is sought and this is given by the sum of preload and eigenvalue. It can be seen from Fig. 8 that the resulting critical moment slightly decreases as the preload increases. Therefore, if the preload is too small, the eigenvalue problem is performed on a model whose state is not representative of the condition prior to buckling and the consequent results are inaccurate. Fig. 8 also shows the mid-section ovalisation (magnified by a factor of $10^{5}$ ) at the end phase (i). To confirm what just stated, one can notice that ovalisation grows more than linearly with the preload and therefore the linear perturbation buckling analysis always overestimates the critical moment until the preload approaches the critical moment.

Additionally, the final predicted buckling pattern is shown in Fig. 8 for four different levels of preload. These images are the contour plots of the nodal total displacement and results were mirrored with respect to the XY plane in order to display the hole pipe. The typical buckling waves cover a pipe axial length shorter and shorter as the preload increases and this is in accordance with real structures' buckling wherein the collapse effects reveal themselves in a somewhat short section of the pipe.

It is opportune to highlight again that results of Sections 4.1, 4.3 and 4.4 refer to the last analysis of each iterative process, that is to say that only simulations with the highest preloads are of interest.

\subsection{Effect of internal pressure on pinched pipe}

The main set of simulations aimed to study the effects of internal pressure on the pinched pipe and quantify its benefits in terms of buckling load increment. Such simulations were performed on models of radius $r=50 \mathrm{~mm}$, length $L=1600 \mathrm{~mm}$ 


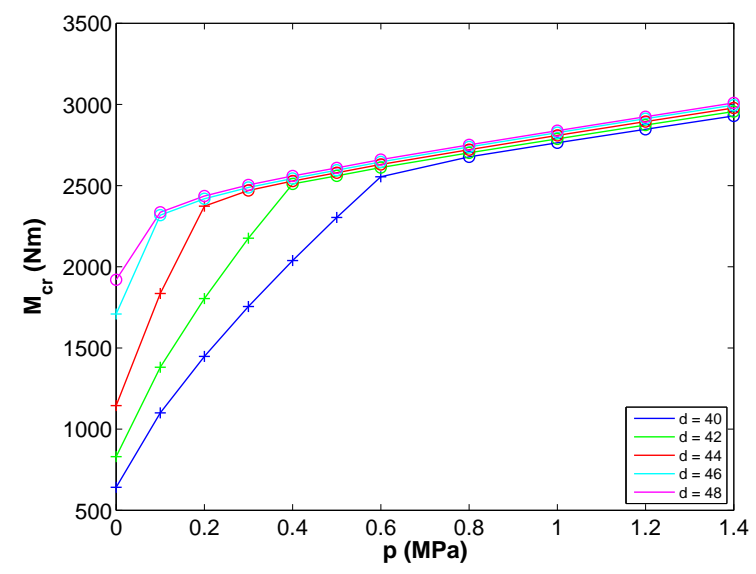

(a) $t=0.25 \mathrm{~mm}$

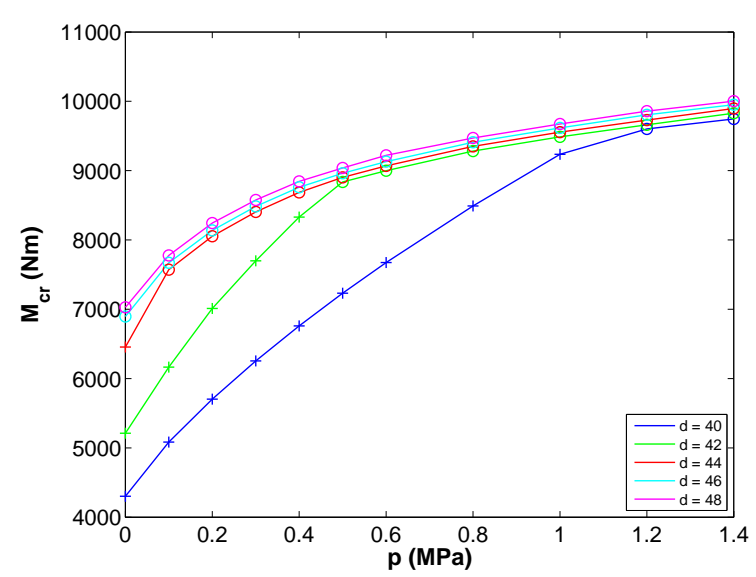

(c) $t=0.50 \mathrm{~mm}$

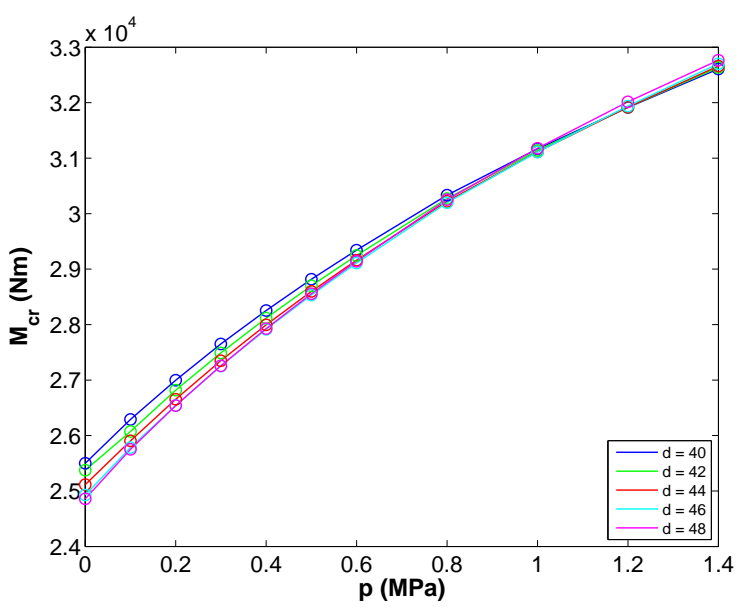

(e) $t=1.00 \mathrm{~mm}$

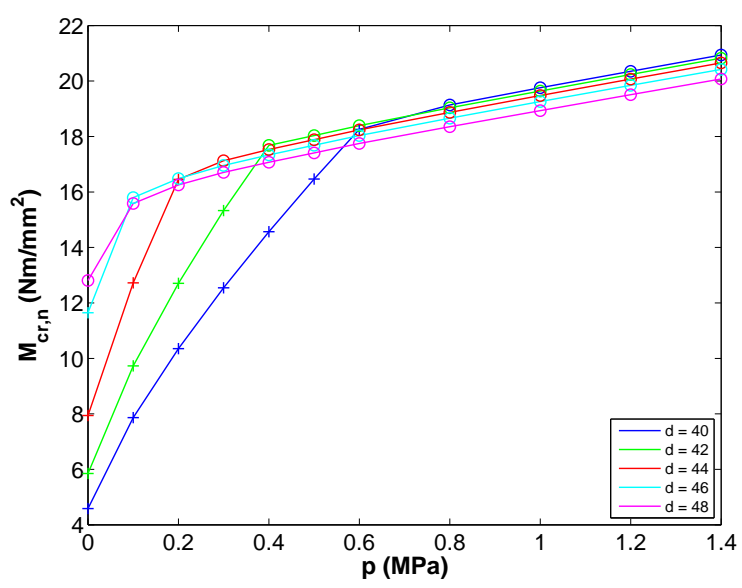

(b) $t=0.25 \mathrm{~mm}$

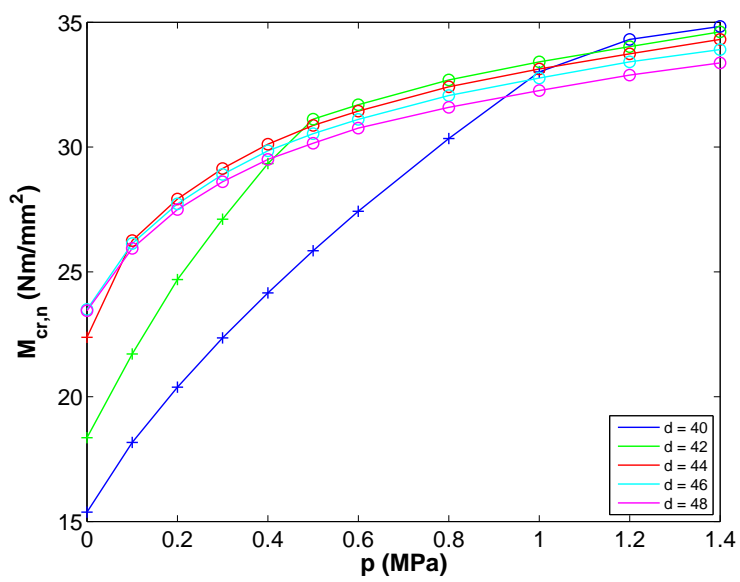

(d) $t=0.50 \mathrm{~mm}$

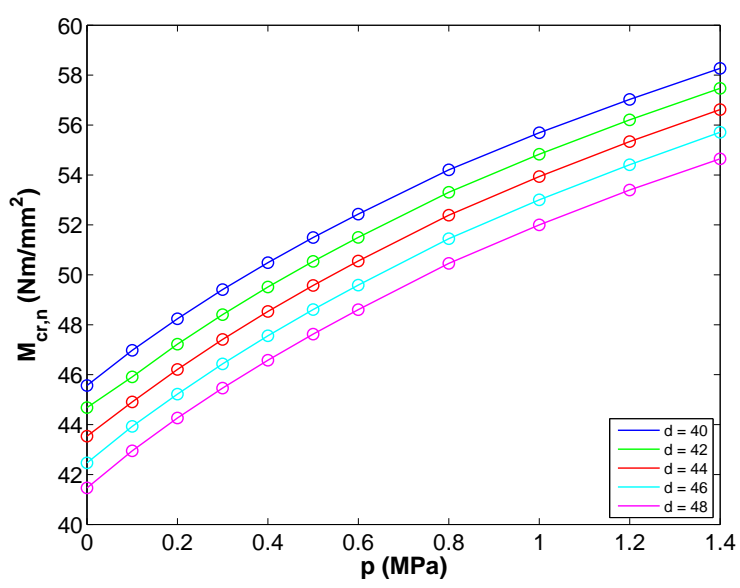

(f) $t=1.00 \mathrm{~mm}$

Figure 9: $(a),(c),(e)$ : critical moment $M_{c r}$ as a function of internal pressure $p .(b),(d),(f)$ : critical moment divided by the crosssection area $M_{c r, n}$ as a function of internal pressure $p$. 
and different values of thickness $t$ and pipe semi-distance $d$. Results are shown in Fig. 9a, 9c and 9e.

Markers in the graphs represent simulation results. There are two types of mark which refer to two different buckling failures. The first type of marker is a 'plus' $(+)$ and indicates that buckling took place on the middle strip of the pinched pipe; the second type is a 'circle' $(\bigcirc)$ and indicates that buckling took place on the bottom side of the pinched pipe. Fig. 10 shows these two different types of buckling.

It can be noted from Fig. 9a, 9c and 9e that pinched pipes with low values of semi-distance $d$ and pressure $p$ tends to buckle on their middle strip whereas high values of semidistance $d$ and pressure $p$ lead to buckling on the pipe. Indeed, a low value of $d$ means a longer strip in the vertical direction (see Fig. 1). When the strip undergoes compression to oppose the cross-section flattening typical of a bent pipe, it can buckle. Hence, for certain pinched pipes, buckling occurs at first on the strip. On the other hand, high values of $d$ denote a pinched pipe with a short strip in the vertical direction which better withstand compression. Moreover, internal pressure causes hoop stress that "stretches" the middle strip. Thus buckling occurs at first on the pipe.

Another consideration on the results shown in Fig. 9a, 9c and $9 \mathrm{e}$ regards the slope change. As long as buckling occurs in the strip, increments of internal pressure lead to significant increments of the critical moment. When the pressure reaches a level sufficient to prevent buckling on the strip, curve slope drops and further increments of internal pressure lead to little increments of the critical moment.

The above simulation results can be presented in a different way to better highlight the performance of each pinched pipe geometry. Thus, it is interesting to plot the critical moment divided by the cross-section area (here referred to as $M_{c r, n}$ ) as a function of internal pressure, shown in Fig. 9b, 9d and 9f for $t=0.25 \mathrm{~mm}, t=0.50 \mathrm{~mm}$ and $t=1.00 \mathrm{~mm}$, respectively. For a certain material (or density), such physical quantity is ultimately an indicator of the strength of the pinched pipe against buckling per unit of linear weight, $\frac{\mathrm{Nm}}{\mathrm{kg} / \mathrm{m}}$. Curves in Fig. 9b, 9d and $9 \mathrm{f}$ have the same trend seen in Fig. 9a, 9c and 9e, however their relative position is different and for high levels of pressure pinched pipes with low values of $d$ become more "efficient" higher critical moment divided by the cross-section area.

\subsection{Pipe semi-distance sensitivity}

The curves shown in Section 4.3 are clearly monotonic, i.e. internal pressurisation is always beneficial in terms of critical buckling moment. Hence, not considering material stress and yield issues, for a given pinched pipe the optimal value of pressure approach infinity. For this reason, it is opportune to present the data in a different form.

The present subsection presents the simulations results as critical moment as a function of geometric variations of the cross-section. In particular, the critical moment divided by the cross-section area $M_{c r, n}$ is plotted as a function of pipe semidistance $d$ for pinched pipes with radius $r=50 \mathrm{~mm}$, length $L=1600 \mathrm{~mm}$ and several values of wall thickness $t$ and internal pressure p. Fig. 11, Fig. 12 and Fig. 13 show the result

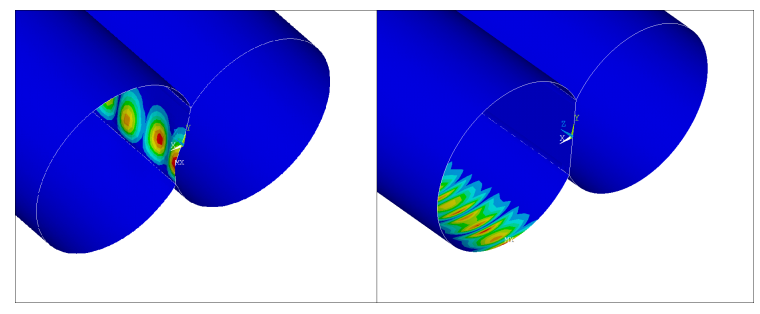

Figure 10: Example of buckling on the strip (left) and on the pipe (right). Contour plots are not axially mirrored therefore the mid-section is shown. Markers + and $\bigcirc$ in the graphs refer to this types of buckling, respectively.

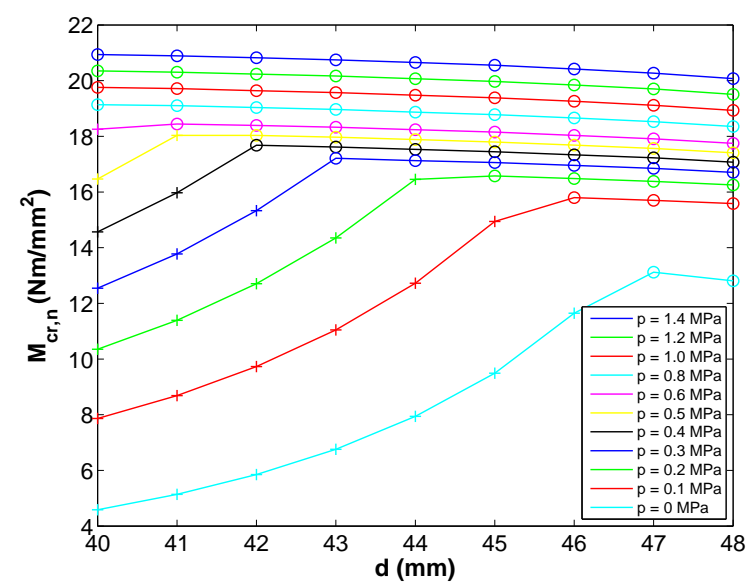

Figure 11: Critical moment divided by the cross-section area $M_{c r, n}$ as a function of pipe semi-distance $d$. Wall thickness $t=$ 0.25 .

for $t=0.25 \mathrm{~mm}, t=0.50 \mathrm{~mm}$ and $t=1.00 \mathrm{~mm}$, respectively. Again, + indicates buckling occurring on the middle strip and $\bigcirc$ indicates buckling occurring on the bottom part of the pinched pipe.

In these graphs it can be noted that once $d$ reaches a value high enough to cause buckling on the pipe, further increments of $d$ are nolonger beneficial. In other words, for a given value of wall thickness $t$ and internal pressure $p$, the optimal crosssection is the one with a value of pipe semi-distance $d$ which balances the two types of buckling failure - buckling on the strip and buckling on the pipe. Furthermore, it appears clear that such optimal value of $d$ decreasing with increaing the internal pressure. For high pressures, the optimal value of $d$ is nomore included in the analysed range, i.e. $d_{\text {optimal }}<40$.

\section{Results remarks}

The previous section showed the results for various pinched pipes subjected to bending moment and internal pressure. This section provides general remarks on the simulation results.

As seen in Fig. 9, pinched pipes take advantage of internal pressure. Larger thicknesses lead to higher increments of critical moment due to pressure. However critical moments with no internal pressure (points along $\mathrm{Y}$ axis) are strongly depen- 


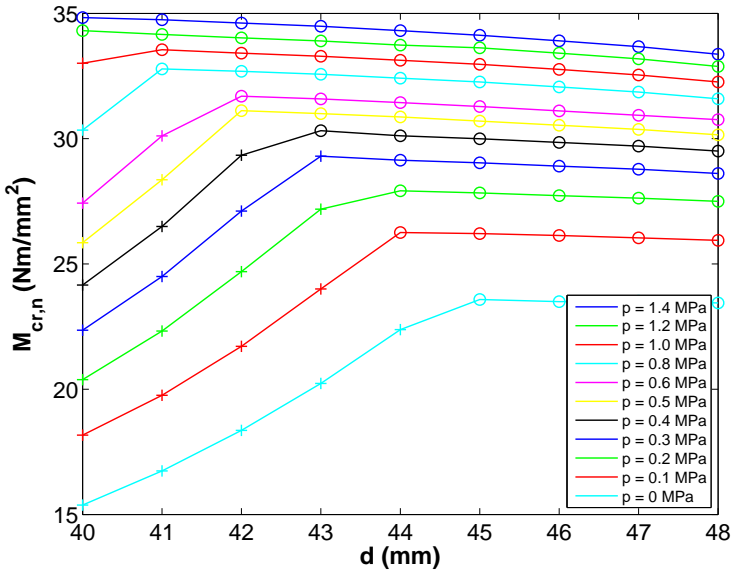

Figure 12: Critical moment divided by the cross-section area $M_{c r, n}$ as a function of pipe semi-distance $d$. Wall thickness $t=$ 0.50 .

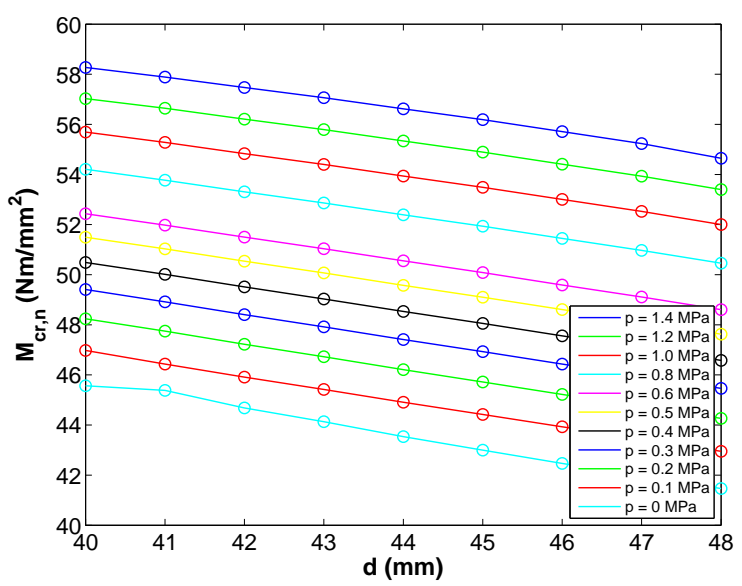

Figure 13: Critical moment divided by the cross-section area $M_{c r, n}$ as a function of pipe semi-distance $d$. Wall thickness $t=$ 1.00 . dent by the the wall thickness. Roughly, it can be said that critical moment with no acting pressure is approximately proportional to the thickness square whereas the curves slope is only slightly more than linearly proportional. As consequence, pinched pipes with thinner walls can exploit internal pressure more and have relatively higher benefits. For $t=0.25 \mathrm{~mm}$, critical moment for pressurised models can be as twice as much the one of the corresponding not pressurised pinched pipes. For $t=1.00 \mathrm{~mm}$, simulation results show increments lower than $30 \%$ of the critical moment for not pressurised pinched pipes. Moreover, the conducted analysis considers a linear material model and therefore plasticity is not taken into account. However, pipes with large wall thickness buckle at bending load corresponding to high stresses, higher than the ones which cause buckling in thinner walls. Internal pressure increases the buckling limit but causes higher stresses too. Then pinched pipes with thin thickness can exploit more the internal pressurisation effects because of their wider margin prior to material plasticity.

It is also worth to notice that the pinched pipe cross-section geometry allows to have high bending load bearing capacity and its structural performance exceeds the one of a single pipe. As reference, a single long pipe with linear material model ( $E$ and $v$ of a steel), $r=50 \mathrm{~mm}$ and $t=0.50 \mathrm{~mm}$ exhibits a critical moment of about $2800 \mathrm{Nm}$, when no internal pressure is acting [15]. A long pinched pipe with the same parameters and $d=45$ is obviously wider but the cross-section height (in the sense of the bending plane) is the same and the cross-section area is less than the double; however it exhibits a critical moment of about $6800 \mathrm{Nm}$.

\section{Applications and manufacturing considerations}

The pinched pipe geometry here presented can be extended from two to a higher number of adjacent pipes. This geometry is expected to withstand high bending loads, especially if exploiting the benefits of internal pressurisation. The tube-shaped nature would also provide good torsion bearing capacity. Such structure is suitable for long span application and internal pressurisation made by air or any other gaseous fluid does not increase the structure weight to any appreciable extent. Hence, its application is desiderable in fields wherein high structural performance and lightness are required, such as aerospace engineering. Particularly, aircraft wings are subjected to high bending loads and appear to be ideal to be made of pinched pipes. A conventional wing structure is made by a "skeleton" of spars (along the wingspan) and ribs (transversal to the spars); around this, panels create the outer airfoil surface. Pinched pipes may lead to an innovative concept wherein the conventional internal wing structure is replaced by a set of adjacent pipes running from the root to the tip of the wing and connected by strips. Fig. 14 shows the concept. Potential advantages would be manifold: high structural performance and opportunity to exploit internal pressurisation. Moreover possibility to vary other structural properties such as wing stiffness by changing pressure level might be investigated.

As herein presented, the proposed structure reveals a certain manufacturing complexity. In particular, it is challenging 

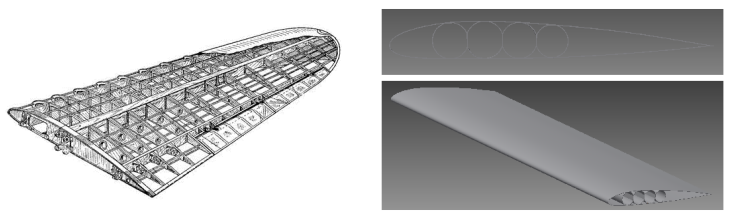

Figure 14: Innovative wing structure concept: conventional structure (left) and internal structure made of pressurised pipes (right).

achieving a cross-section geometry as the one shown in Fig. 1. However, slight modifications can allow an easier manufacturing process. For example, the pinched pipe can be made by two $D$-shape cross-section pipes welded together. The optimisation analysis could be quickly adjusted to take into account the resulting double thickness of the middle strip - although residual stress may need to be considered. Moreover, the use of composite can even avoid the need of welding since parts can be joined by means of the curing process. In the case of composite, the initial investment related to the mould manufacturing is paid back by the high freedom in shape design. On this regard, it is worth to notice that the pressurised pipes can be built in such a way that the cross-section decreases in size along the span. As first advantage, some manufacturing procedures could benefit from this "taper angle" - easier mould extraction in cases of inner mould. Additionally, a variable cross-section is structurally more efficient in applications as the one shown in Fig. 14 since a wing is mostly loaded at the root while the wing tip can be thinner and lighter.

\section{Conclusion}

The present paper proposes a novel stiffener for bending loads referred to as pinched pipe. This is proved to have high bending load bearing capacity. The long known benefits of pressure against buckling phenomenon are confirmed by this study. The presented structure seems to well exploit internal pressurisation and the consequent increments of critical bending load are significant. Low values of pressure $(\approx 0.3 \mathrm{MPa})$ are generally sufficient to lead to high critical moment increments. The pinched pipe presents two different buckling modes and, for a certain geometry, the internal pressure level can determine which one will occur.

Results show that pinched pipe exhibits higher performance than the one of a single pipe in terms of bending moment bearing capacity over cross-section area ratio. Given a certain pressure level, the cross-section geometry should be such that the two different buckling modes (on strip and on pipe) tend to occur simultaneously, in order to maximise the performance. This can be done by setting the right value of the here so-called $d$ parameter.

The analysed geometry is the base case and appears to be challenging from the manufacturing point of view. Nevertheless it opens the way for a series of similar structures whose performance may justify the manufacturing effort. A possible application of the pinched pipe structure has been presented.
Further studies are desiderable on this matter. Moreover, optimisation of the cross-section geometry involving studies with two different values of wall thickness for strips and pipes may further increase the structural performance.

\section{Acknowledgements}

This work is funded by the European Commission under the project titled INNOVATE, The systematic Integration of Novel Aerospace Technologies, FP7 project number 608322 which is part of the FP7-PEOPLE-2013-ITN call. The authors would like to gratefully acknowledge the Marie Curie Innovative Doctoral Programme for the financial support.

\section{References}

[1] Bryan G. On the stability of a plane plate under thrusts in its own plane, with applications to the buckling of the sides of a ship. Proceedings of the London Mathematical Society 1890;1(1):54-67.

[2] Timoshenko SP, Gere JM. Theory of elastic stability 1961;.

[3] Brazier L. On the flexure of thin cylindrical shells and other" thin" sections. Proceedings of the Royal Society of London Series A 1927;116(773):104-14.

[4] Chen W, Sohal I. Cylindrical members in offshore structures. ThinWalled Structures 1988;6(3):153-285.

[5] Hauch SR, Bai Y. Bending moment capacity of pipes. Journal of Offshore Mechanics and Arctic Engineering 2000;122(4):243-52.

[6] Hilberink A. Mechanical behaviour of lined pipe. Ph. D. Thesis, Delft Technical University, ISBN 978-94-6186-012-5; 2011.

[7] Mørk K, Spiten J, Torselletti E, Ness O, Verley R. The superb project and dnv'96: Buckling and collapse limit state. In: Proceedings of the International Conference on Offshore Mechanics and Arctic Engineering. American Society of Mechanical Engineers; 1997, p. 79-90.

[8] Chwalla E. Reine biegung schlanker, dünnwandiger rohre mit gerader achse. ZAMM-Journal of Applied Mathematics and Mechanics/Zeitschrift für Angewandte Mathematik und Mechanik 1933;13(1):48-53.

[9] Hutchinson J. Axial buckling of pressurized imperfect cylindrical shells. AIAA Journal 1965;3(8):1461-6.

[10] Crate H, Batdorf S, Baab GW. The effect of internal pressure on the buckling stress of thin-walled circular cylinders under torsion. Tech. Rep.; DTIC Document; 1944.

[11] Robertson A, Li H, Mackenzie D. Plastic collapse of pipe bends under combined internal pressure and in-plane bending. International Journal of Pressure Vessels and Piping 2005;82(5):407-16.

[12] Mourad HM, Younan MY. Limit-load analysis of pipe bends under outof-plane moment loading and internal pressure. Journal of pressure vessel technology 2002;124(1):32-7.

[13] Polenta V, Garvey S, Chronopoulos D, Long A, Morvan H. Effects of pipe curvature and internal pressure on stiffness and buckling phenomenon of circular thin-walled pipes. International Journal of Mechanical, Aerospace, Industrial and Mechatronics Engineering 2015;9(2):27882.

[14] Yudo H, Yoshikawa T. Buckling phenomenon for straight and curved pipe under pure bending. Journal of Marine Science and Technology 2014;.

[15] Polenta V, Garvey S, Chronopoulos D, Long A, Morvan H. Optimal internal pressurisation of cylindrical shells for maximising their critical bending load. Thin-Walled Structures 2015;87:133-8.

[16] ANSYS Academic Research, Release 15.0, Help System, Mechanical APDL Structural Analysis Guide, 7. Buckling Analysis; 2013.

[17] ANSYS Academic Research, Release 15.0, Help System, Mechanical APDL Theory Reference, 15.8. Linear Perturbation Analysis; 2013.

[18] Guarracino F, Walker A, Giordano A. Effects of boundary conditions on testing of pipes and finite element modelling. International Journal of Pressure Vessels and Piping 2009;86(2):196-206.

[19] Love AEH. A treatise on the mathematical theory of elasticity; vol. 1. Cambridge University Press; 2013. 